\title{
Erratum
}

\section{Characters and $\boldsymbol{K}$-theory of discrete groups}

\author{
Alejandro Adem \\ Invent. math. 114, 489-514 (1993)
}

It has been pointed out to me that proposition 2.7 is incorrect. There are examples of arithmetic groups for which this fails. I am grateful to O. Gabber and J-P. Serre for pointing this out to me.

The main results in the paper are unaffected by this. Corollary 4.5 can be proved without making use of this result. First note that the additive decomposition used in 5.8 at the level of components is obtained as follows: if $\gamma \in \operatorname{Tors}(\Gamma)$, apply the restriction from $K_{\Gamma}^{*}(X)$ to $K_{\langle\gamma\rangle}^{*}\left(X^{\langle\gamma\rangle}\right) \cong R(\langle\gamma\rangle)$, followed by the map $R(\langle\gamma\rangle) \rightarrow \mathbb{C}$ given by taking the generator to the corresponding finite root of unity. From the proof of the decomposition in 5.8 (see $[\mathrm{K}]$ ), we infer that the sum of these compositions over distinct conjugacy classes induces a surjection $K_{\Gamma}^{*}(X) \otimes \mathbb{C} \rightarrow(\mathbb{C})^{n(\Gamma)}$, factoring through the restriction map to $R_{\mathscr{F}}(\Gamma) \otimes \mathbb{C}$ (identified as a character ring according to 2.8 ). Hence the ring map induced by the restrictions $K_{\digamma}^{*}(X) \otimes \mathbb{Q} \rightarrow R_{\mathscr{F}}(\Gamma) \otimes \mathbb{Q}$ is a surjection.

This is actually a special case of a result in equivariant $K$-theory. Namely if $Y$ is a compact $G-C W$ complex, $G$ a finite group, let $\mathscr{F}_{G}(Y)$ denote the ring of compatible families of locally constant maps $Y^{H} \rightarrow R(H)$, where $H$ is a subgroup of $G$ (see [Q] for details). This can be identified with a limit of representation rings taken over the category of pairs $(H, c)$ where $c \in \pi_{0}\left(Y^{H}\right)$, with the usual morphisms à la Quillen. Then what we have is that the natural map induced by restrictions $K_{G}^{*}(Y) \rightarrow \mathscr{F}_{G}(Y)$ is a rational surjection. In our case we let $Y=X / \Gamma^{\prime}, G=\Gamma / \Gamma^{\prime}$, and as pointed out by Quillen, $\mathscr{F}_{G}(Y) \cong R_{\mathscr{F}}(\Gamma)$, and also $K_{G}^{*}(Y) \cong K_{\Gamma}^{*}(X)$, from which the result above follows. The point here is that the fixed-point data are necessary to distinguish conjugacy classes of elements of finite order in $\Gamma$, and this is part of the known rational decomposition of equivariant $\mathbf{K}$-theory.

I would also like to take this opportunity to point out that in expression 4.7 the indices $p, q$ should be exchanged. Finally I would like to mention that C.N. Lee has proved [L] that if $f: \Gamma_{1} \rightarrow \Gamma_{2}$ is a homomorphism between groups satisfying our hypotheses which induces a $\mathbb{Z}_{p}$ homology equivalence, then the two groups in fact have isomorphic posets of finite $p$-subgroups, which implies in particular that $R_{\mathscr{F}(p)}\left(\Gamma_{1}\right) \cong R_{\mathscr{F}(p)}\left(\Gamma_{2}\right)$. 


\section{References}

[K] N. Kuhn; Character Rings in Algebraic Topology. In: London Math. Soc. LNS 139, Cambridge U. Press

[L] C.N. Lee: Stable Homotopy Type of the Classifying Space of Virtually Torsion-Free Groups (Preprint 1993)

[Q] D. Quillen: The Spectrum of an Equivariant Cohomology Ring I \& II. Ann. Math. 94, $549-602(1971)$ 